G. F. Dumont

P. Descroix

\title{
La fécondité de la France par rapport à celle des pays
} industriels (1963-1985)

In: Population, 44e année, n², 1989 pp. 441-444.

Citer ce document / Cite this document :

Dumont G. F., Descroix P. La fécondité de la France par rapport à celle des pays industriels (1963-1985). In: Population, 44e année, $n^{\circ} 2,1989$ pp. 441-444.

http://www.persee.fr/web/revues/home/prescript/article/pop_0032-4663_1989_num_44_2_3467 
F. Dirfal. O. Barbary, A. Michel. - Intégration des données de télédétection dans un sustème d'information géographique : suivi de la morphologie et de la démographie d'une" iille. Rapport intermédiaire, ORSTOM. Département D. Avril 1986.

F. Dereal: O. Barbary. A. Michel. - "Intégration des données de télédétection dans un système d’information géographique : suivi de la morphologie et de la démographie d'une ville ". Rapport final, Volume 1 : Synthèse. Volume 2 : Annexes. ORSTOM, Département D, août 1987.

A. Michel, J.-M. Eberhard, B. Lortic. F. Dureal. - « Lutilisation de la télédétection pour lobservation des populations urbaines. Une recherche en cours a Quito (Equateur) ". Colloque SPOT, Bogota, 16-20 novembre 1987.

O. BArbary. - "Mise au point de plans de sondage pour l'estimation de caractères démographiques à Marseille. Evaluation des précisions permises par l'emploi de diverses techniques de sélection, de stratification et destimation ". EHESS-ORSTOM. document interne, ORSTOM. Département SDU U.R. : Sistèmes d'accumulation des hommes el des richesses. janvier 1988.

O. BARBARY, en collaboration avec F. DUREAl; et A. MichtL. - «Définition et mise en cuvre d'un plan de sondage aréolaire sur image SPOT pour une enquête démographique à Quito (Equateur) ». Document interne, ORSTOM. Département SDU U.R. : "Syste. mes d'accumulation des hommes et des richesses ". mai 1988.

F. DereAl, J.-P. DeChEMIX, Département SDU. - Lutilisation de limagerie SPOT pour lobseriation démographique e'n milieu urhain. Rapport final. Convention CNESORSTOM. avril 1988.

F. DLREAL: - Eléments de calcul des coùts d'enquête à Quito. ORSTOM. Département SDU U.R. 15, note interne, Quito, Août 1988.

F. DlReal. - «A propos de l'analyse des systèmes résidentiels - Présentation de l'enquête Migrations réalisée à Quito (Equateur) en décembre 1987 ". Colloque Migralion.s, changements sociaux et développement, ORSTOM, 20-22 septembre 1988, 16 pages.

F. Direau, O. Barbary, B. Lortic, A. Michli. - "Utiliser la télédétection spatiale pour produire rapidement des données démographiques en ville. L'exemple de Quito (Equateur)". Colloque Gestion urbaine ef développement". Lyon, 20-22 septembre 1988, 18 pages.

O. BARBARY. - Sondages aréolaires pour l'estimation de données démographiques en milieu urhain. Essai de définition d'une méthode de collecte intégrant linformation satellitaire. Application aux villes des pałs en développement. Thèse de doctorat, Ecole des Hautes Etudes en Sciences Sociales, Direction de M. BARBUT, 7/12/1988.

F. DUREAL. - A propos du traitement informatique des données localisées. Une expérience en cours : télédétection el obsenation des populations urbaines. ORSTOM, Département D. 1988.

\section{LA FÉCONDITÉ DE LA FRANCE PAR RAPPORT Ä CELLE DES PAYS INDUSTRIELS (1963-1985)}

A partir de 1944, la France participe à la croissance de la fécondité qui se manifeste alors en Europe. Puis vers 1964, en France comme dans la plupart des pays industriels à faible fécondité précoce, commence une baisse de fécondité dont l'allure est étonnamment régulière, une certaine stabilisation intervenant après 1978. Or au cours de cette période, la France se singularise par une chute moins rapide de ses indices synthétiques de fécondité (ISF), et par leur stabilisation à un niveau relativement élevé.

Quel est le nombre de naissances supplémentaires constatées en France de 1963 à 1985 du fait de cette allure particulière des ISF ? 


\section{Choix des pays de référence}

et des courbes caractéristiques
Francisco Muñoz-Pérez a publié "il un tableau donnant l'ISF des femmes de 22 pays européens pour chaque année de 1960 à 1985. Les pays que nous avons retenus pour nos comparaisons sont ceux qui manifestent une évolution de leur ISF parallèle dans le temps à celle qui est constatée en France : Angleterre-Galles, Autriche, Belgique, Danemark, Norvège, Pays-Bas, R.F. Allemagne, Suède et Suisse. On peut leur ajouter les États-Unis et le Canada, ce qui fait onze pays.

Les courbes d'évolution des ISF de ces pays, et surtout leur moyenne annuelle, ont dans l'ensemble l'allure des courbes anti-logistiques proposée par Marc Artzrouni ' 2 . Mais il est dans l'espèce impossible de calculer l'équation de courbes de cette forme, étant donné leur sensibilité aux irrégularités.

TABLEAL 1. - Évolution dES INDICES SPECIFIQUES DE FÉCONDITÉ DES FEMMES EN ElROPE ENTRE 1964 ET 1985

\begin{tabular}{|c|c|c|c|c|c|}
\hline Pays & Années & Moyenne ISF & $\begin{array}{c}\text { Variation ISF } \\
\text { annuelle }\end{array}$ & Corrélation & $\begin{array}{l}\text { Variation en } \% \\
\text { de la moyenne }\end{array}$ \\
\hline Angleterre & $\begin{array}{l}1963-85 \\
1964-77\end{array}$ & $\begin{array}{l}2,15 \\
2,31\end{array}$ & $\begin{array}{c}-0,059 \\
-0,10\end{array}$ & $\begin{array}{l}-0,918 \\
-0,993\end{array}$ & $\begin{array}{l}-2,74 \\
-4,41\end{array}$ \\
\hline Autriche & $\begin{array}{l}1963-85 \\
1963-79\end{array}$ & $\begin{array}{l}2,05 \\
2,20\end{array}$ & $\begin{array}{l}-0,065 \\
-0,09\end{array}$ & $\begin{array}{l}-0,952 \\
-0,989\end{array}$ & $\begin{array}{l}-3,17 \\
-3,95\end{array}$ \\
\hline Belgique & $\begin{array}{l}1963-85 \\
1964-75\end{array}$ & $\begin{array}{l}1,99 \\
2,24\end{array}$ & $\begin{array}{l}-0,057 \\
-0,08\end{array}$ & $\begin{array}{l}-0,969 \\
-0,988\end{array}$ & $\begin{array}{l}-2.59 \\
-3,71\end{array}$ \\
\hline Canada & $\begin{array}{l}1963-85 \\
1960-74\end{array}$ & $\begin{array}{l}2,20 \\
2,86\end{array}$ & $\begin{array}{l}-0,081 \\
-0,17\end{array}$ & $\begin{array}{l}-0,895 \\
-0,932\end{array}$ & $\begin{array}{l}-3,69 \\
-5,80\end{array}$ \\
\hline Danemark & $\begin{array}{l}1963-85 \\
1966-83\end{array}$ & $\begin{array}{l}1,91 \\
1,85\end{array}$ & $\begin{array}{l}-0,06 \\
-0,06\end{array}$ & $\begin{array}{l}-0,965 \\
-0,955\end{array}$ & $\begin{array}{l}-3,08 \\
-3,13\end{array}$ \\
\hline États-Unis & $\begin{array}{l}1963-83 \\
1960-73\end{array}$ & $\begin{array}{l}2,15 \\
2.76\end{array}$ & $\begin{array}{l}-0,06 \\
-0,13\end{array}$ & $\begin{array}{l}-0,874 \\
-0,985\end{array}$ & $\begin{array}{l}-2,88 \\
-4,86\end{array}$ \\
\hline France & $\begin{array}{l}1963-85 \\
1963-78\end{array}$ & $\begin{array}{l}2,24 \\
2,40\end{array}$ & $\begin{array}{l}-0,056 \\
-0,08\end{array}$ & $\begin{array}{l}-0,944 \\
-0,979\end{array}$ & $\begin{array}{l}-2,50 \\
-3,33\end{array}$ \\
\hline Norvège & $\begin{array}{l}1963-85 \\
1963-77\end{array}$ & $\begin{array}{l}2,22 \\
2,49\end{array}$ & $\begin{array}{l}-0,072 \\
-0.09\end{array}$ & $\begin{array}{l}-0,970 \\
-0,983\end{array}$ & $\begin{array}{l}-3,25 \\
-3,65\end{array}$ \\
\hline Pays-Bas & $\begin{array}{l}1963-85 \\
1964-75\end{array}$ & $\begin{array}{l}2,11 \\
2,48\end{array}$ & $\begin{array}{l}-0,088 \\
-0,14\end{array}$ & $\begin{array}{l}-0,945 \\
-0,979\end{array}$ & $\begin{array}{l}-4,18 \\
-5,56\end{array}$ \\
\hline RF Allemagne & $\begin{array}{l}1963-85 \\
1966-79\end{array}$ & $\begin{array}{l}1,79 \\
1,81\end{array}$ & $\begin{array}{l}-0,066 \\
-0,10\end{array}$ & $\begin{array}{l}-0,929 \\
-0,955\end{array}$ & $\begin{array}{l}-3,69 \\
-5,52\end{array}$ \\
\hline Suède & $\begin{array}{l}1963-85 \\
1964-78\end{array}$ & $\begin{array}{l}1,90 \\
1,98\end{array}$ & $\begin{array}{l}-0,038 \\
-0,06\end{array}$ & $\begin{array}{l}-0,907 \\
-0,962\end{array}$ & $\begin{array}{l}-2,00 \\
-3,02\end{array}$ \\
\hline Suisse & $\begin{array}{l}1963-85 \\
1964-76 \\
\end{array}$ & $\begin{array}{l}1,90 \\
2,11 \\
\end{array}$ & $\begin{array}{l}-0,059 \\
-0,10 \\
\end{array}$ & $\begin{array}{l}-0,935 \\
-0,999 \\
\end{array}$ & $\begin{array}{l}-3,10 \\
-4,60 \\
\end{array}$ \\
\hline $\begin{array}{l}\text { Ensemble sans } \\
\text { la France }\end{array}$ & $\begin{array}{l}1963-85 \\
1963-78\end{array}$ & $\begin{array}{l}2,03 \\
2,22\end{array}$ & $\begin{array}{l}-0,064 \\
-0,092\end{array}$ & $\begin{array}{l}-0,950 \\
-0,994 \\
\end{array}$ & $\begin{array}{l}-3,15 \\
-4,14\end{array}$ \\
\hline
\end{tabular}

(1) Thèse de doctorat soutenue le 7 décembre 1988 à l'École des Hautes Études en Sciences Sociales sous la direction de Marc Barbut.

(2) J.-J. Droesbeke. B. Fichet. Ph. Tassi. Les sondages, Association pour la statistique et ses utilisations. 1987. Economica. 
Toutefois, l'examen des graphiques 1 de Muñoz-Pérez fait ressortir des points d'inflexion qui permettent de découper les courbes en un certain nombre de segments. Pour chaque courbe entière de 1963 à 1985 , puis pour le principal segment entre les dates caractéristiques, variables suivant les pays, de l'effondrement constaté à partir du premier tiers des années 1960 , nous avons calculé les droites de régression linéaire, les coefficients de corrélation et les moyennes de l'ISF. Nous y avons ajouté le pourcentage de variation de l'ISF, obtenu en divisant le coefficient de régression linéaire par la moyenne de l'indice entre les dates considérées (tableau 1).

On voit qu'au cours d'une période qui dure de 12 à 15 ans, la fécondité des femmes européennes des pays considérés a diminué en moyenne de 0,09 point, à l'allure de $4,1 \%$, par an. Pour la France, cette diminution a été un peu moins rapide : 0,08 point et $3,3 \%$ par an.

Le graphique ci-après donne, pour la France et pour les onze autres pays examinés, les valeurs année par année entre 1963 et 1985 ainsi que la droite de régression entre 1963 et 1978 . Il fait ressortir le niveau constamment plus élevé de la courbe pour la France et la relative stabilisation des indices après 1978.

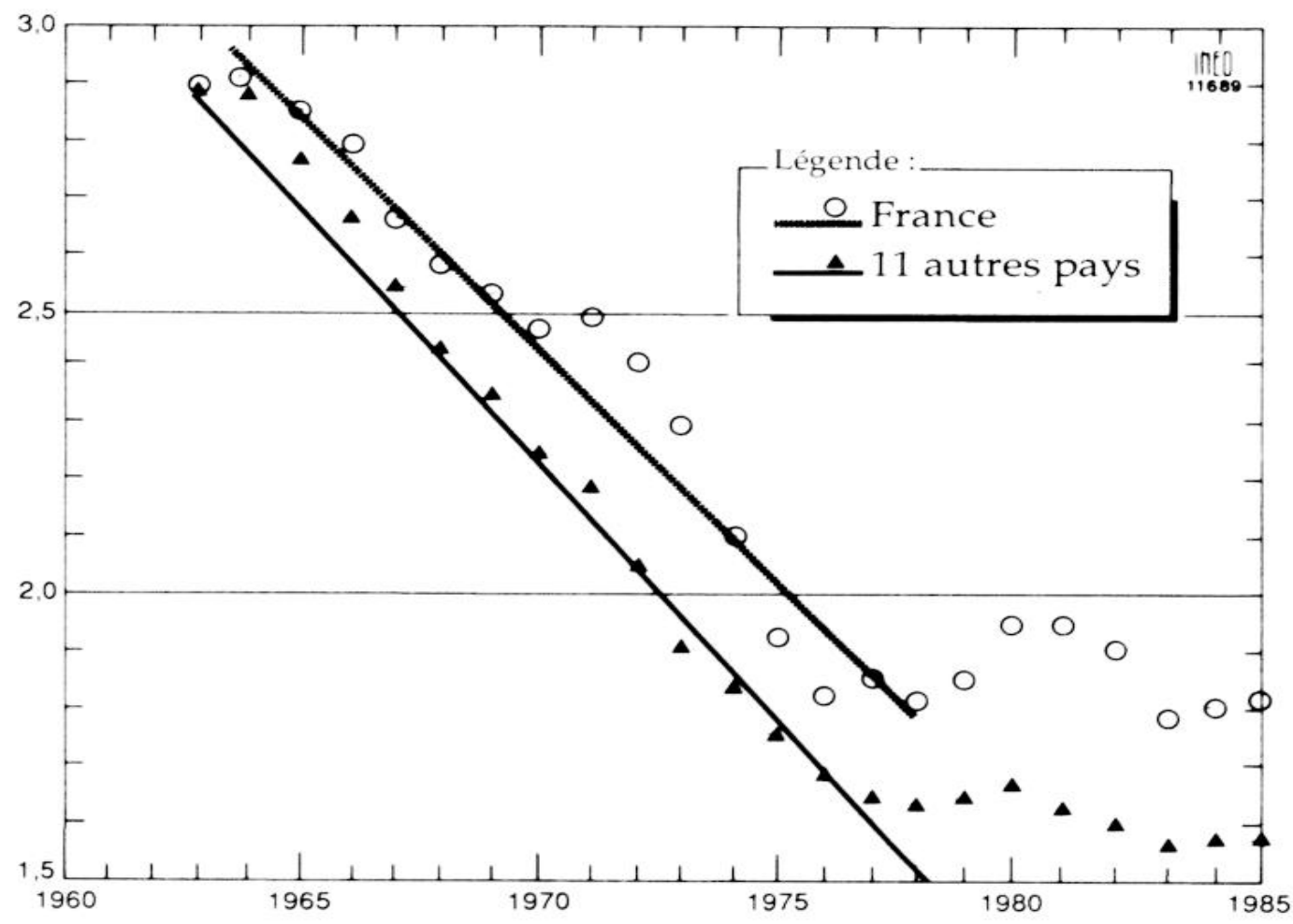

La surfécondité française

Pour obtenir le différentiel de la fécondité française par rapport à celle des pays considérés, on établit le rapport, par année, de la moyenne des ISF des onze pays de comparaison sur l'ISF de la France pour cette année-là. En le multipliant par le nombre de naissances enregistré en France pour la même année $N$ on obtient un chiffre de naissances corrigé $N C$, d'où le différentiel de naissances $d=N C-N$.

De 1960 à 1962, ce différentiel était négatif. En 1963, il devient modestement positif (3000), et ne cesse de grandir jusqu'à 1973 où il atteint son maximum, 
142000 . Ensuite il s'effondre en trois ans à 53650 en 1976, avant de remonter jusqu'à un deuxième maximum de 131500 en 1981 et fluctuer ensuite entre 75000 et 100000 environ.

La somme des naissances enregistrées en France de 1963 à 1985 est :

$$
S=18612000
$$

et le cumul des différentiels de naissances pour la même période par rapport aux pays européens donne :

$$
D=1800200
$$

d'où le rapport :

$$
D / S=0,096
$$

Entre 1963 et 1985 , la France a compté près de $10 \%$ de naissances de plus que si l'indice synthétique de la fécondité de ses femmes avait évolué de la même façon que dans les onze pays industriels où l'évolution de cet indice est parallèle à l'évolution du sien.

\section{Explications démographiques}

Une première explication de cette différence de comportement peut être recherchée dans la densité de population des pays intéressés. Mais elle n'est pas valable, l'ISF de l'Allemagne par exemple ayant varié plus vite que celui de la Belgique, plus densément peuplée.

Une autre explication pourrait être le comportement des femmes immigrées. Elle est d'autant plus tentante que, lors de la période de stabilisation (environ 1977-85) qui marque la fin des années considérées, la valeur moyenne de l'ISF en Europe étant de 1,62 , cette moyenne est particulièrement élevée dans trois pays où l'immigration en provenance du tiers monde est forte: la France (moyenne 1977-86:1,86, pratiquement stable), l'Angleterre (moyenne 1977-86:1,78, stable) et les États-Unis (moyenne 1973-86:1,80, ascendante).

En France les immigrées les plus fécondes sont les maghrébines. Mais ce n'est guère que depuis 1974 (loi permettant le regroupement des familles) que les maghrébines ont commencé à arriver en nombre. De toute façon, les données disponibles ne permettent pas de quantifier l'importance de ce facteur explicatif.

Une explication plus satisfaisante peut être qu'en France, sans doute en raison de la prise de conscience plus ancienne de l'importance de l'évolution de la natalité, il y a un désir d'enfants que des lois comme le Code de la Famille de 1939 et les textes ultérieurs rédigés dans le même esprit ont favorisé.

\section{Gérard-François Dumont et Pierre Descrolx.}

